\title{
Erratum: Constraints on Low-Energy Effective Theories from Weak Cosmic Censorship [Phys. Rev. Lett. 126, 031102 (2021)]
}

\author{
Baoyi Chen, Feng-Li Lin๑, Bo Ning, and Yanbei Chen \\ (Received 20 February 2021; published 18 March 2021)
}

DOI: 10.1103/PhysRevLett.126.119903

As pointed out by Ref. [1], our $A_{a}$ in the original Eq. (6) did not satisfy the equation of motion. This mistake arose because, when solving for $F_{a b}$, we had inconsistently neglected the difference between the background and the perturbed covariant derivatives. The correct solution should instead be

$$
A_{t}=-\frac{q}{r}-\frac{q^{3}}{5 r^{5}} \times\left[c_{2} \kappa^{2}+4 c_{3} \kappa^{2}+10 c_{4} \kappa+c_{5} \kappa-c_{6} \kappa\left(9-\frac{10 m r}{q^{2}}\right)-16 c_{7}-8 c_{8}\right] .
$$

This leads to a corrected $c_{0}^{\prime}$ in Eq. (12):

$$
c_{0}^{\prime}=c_{2}+4 c_{3}+\frac{c_{5}}{\kappa}+\frac{c_{6}}{\kappa}+\frac{4 c_{7}}{\kappa^{2}}+\frac{2 c_{8}}{\kappa^{2}}=c_{0} .
$$

Using this new expression, and continuing on with the rest of the discussions in the original paper, we conclude that the weak cosmic censorship conjecture (WCCC) will always be valid for in-falling matter that satisfy the weak energy condition. In this way, no constraints can be imposed on the coefficients $c_{i}$ ' by imposing the WCCC. This is exactly opposite to our original conclusion but is still interesting in the following two aspects: (i) for near-extremal black holes, our result calls for a check of WCCC at the quartic order for low-energy effective theories (EFTs) considered in our paper; (ii) for extremal black holes, one might wonder whether WCCC holds for all other EFTs in general, i.e., whether $c_{0}^{\prime}$ coincides with $c_{0}$ for a reason.

While more technical work is needed to clarify (i), definite conclusions can be drawn for (ii). More specifically, one can prove that WCCC is preserved for nonrotating extremal black holes in all $n$-dimensional diffeomorphism-covariant theories of gravity and $U(1)$ gauge field; this has also been done by Ref. [1].

As has been correctly argued in the paper, in order for matter to fall into the black hole we must have

$$
\delta M-\Phi_{H} \delta Q \geq 0
$$

assuming that the infalling matter obeys the null energy condition. On the other hand, the condition for the extremal solution to not become singular, i.e, to preserve the WCCC, is given by

$$
\delta M-\left(\frac{d M}{d Q}\right)_{\mathrm{ext}} \delta Q \geq 0,
$$

where $(d M / d Q)_{\text {ext }}$ is the derivative taken along the extremal trajectory in the $(M, Q)$ space. Note that this relation is the generic form of Eq. (14) in our Letter. Here we have also assumed that $(d M / d Q)_{\text {ext }}>0$ and that nonextremal black holes have $M>M_{\text {ext }}(Q)$. Some examples violating these assumptions is discussed in [2]; however, the associated physical implication is obscure.

We then write the first law of black hole thermodynamics for nonextremal black holes,

$$
\delta M=T \delta S+\Phi_{H} \delta Q
$$

where $S$ in the entropy of the black hole. As we approach the extremal solution, we have $T \rightarrow 0$, and

$$
\left(\frac{d M}{d Q}\right)_{\mathrm{ext}}=\Phi_{H}
$$


This relation can be verified explicitly for the quartic EFTs studied in this paper. Because of (3) and (6), (4) always holds and concludes our proof. Obviously, the above proof will not work for near-extremal cases because (6) does not hold.

In conclusion, no matter how we change the action of the EFTs, the condition for the matter falling into the extremal black hole always coincides with the condition for WCCC. Thus, WCCC for extremal black holes will not constrain the form of low-energy EFTs.

[1] J. Jiang, A. Sang, and M. Zhang, arXiv:2101.10172.

[2] J. Jiang and M. Zhang, Phys. Rev. D 102, 084033 (2020). 